\title{
Gestational Diabetes Mellitus Risk score: A practical tool to predict Gestational Diabetes Mellitus risk in Tanzania
}

Anna Patrick Nombo (Sokoine Univeristy of Agriculture, Department of Food Technology, Nutrition and Consumer Sciences, P.O Box 3006, Morogoro, Tanzania).

Dr. Akwilina Wendelin Mwanri (Sokoine University of Agriculture, Department of Food Technology, Nutrition and Consumer Sciences, P.O Box 3006, Morogoro, Tanzania).

Dr. Elske M. Brouwer-Brolsma (Wageningen University and Research centre, Division of Human Nutrition, P. O. Box 6703HD Wageningen, Wageningen, The Netherlands)

Dr Kaushik L Ramaiya (Shree Hindu Mandal Hospital, P O Box 581, Dar es Salaam, Tanzania)

Prof. Edith Feskens (Wageningen University and Research centre, Division of Human Nutrition, P.O Box 17, 6700AA Wageningen, The Netherlands)

\section{Abstract}

Background: Universal screening for hyperglycemia during pregnancy may be in-practical in resource constrained countries. Therefore, the aim of this study was to develop a simple, non-invasive practical tool to predict undiagnosed Gestational diabetes mellitus (GDM) in Tanzania.

Methods: We used cross-sectional data of 609 pregnant women, without known diabetes, collected in six health facilities from Dar es Salaam city (urban). Women underwent screening for GDM during ante-natal clinics visit. Smoking habit, alcohol consumption, preexisting hypertension, birth weight of the previous child, high parity, gravida, previous caesarean section, age, MUAC $\geq 28 \mathrm{~cm}$, previous stillbirth, haemoglobin level, gestational age (weeks), family history of type 2 diabetes, intake of sweetened drinks (soda), physical activity, vegetables and fruits consumption were considered as important predictors for GDM. Multivariate logistic regression modelling was used to create the prediction model, using a cut-off value of 2.5 to minimise the number of undiagnosed GDM (false negatives).

Results: Mid-upper arm circumference (MUAC) $\geq 28 \mathrm{~cm}$, previous stillbirth, and family history of type 2 diabetes were identified as significant risk factors of GDM with a sensitivity, specificity, positive predictive value, and negative predictive value of $69 \%, 53 \%, 12 \%$ and $95 \%$, respectively. Moreover, the inclusion of these three predictors resulted in an area under the curve $(A \cup C)$ of $0.64(0.56-0.72)$, indicating that the current tool correctly classifies $64 \%$ of high risk individuals.

Conclusion: The findings of this study indicate that MUAC, previous stillbirth, and family history of type 2 diabetes significantly predict GDM development in this Tanzanian population. However, the developed non-invasive practical tool to predict undiagnosed GDM only identified 6 out of 10 individuals at risk of developing GDM. Thus, further 
development of the tool is warranted, for instance by testing the impact of other known risk factors such as maternal age, pre-pregnancy BMI, hypertension during or before pregnancy and pregnancy weight gain.

Keywords: Gestational diabetes mellitus, Risk score, prediction model, Tanzania.

\section{Introduction}

During the last decade the prevalence of Gestational Diabetes Mellitus (GDM) considerably increased ranging from 1 to $20 \%$ of pregnancies, depending on the studied population, screening approaches applied, and diagnostic criteria [1-3]. In Tanzania, the overall prevalence of GDM is averaged to be about $6 \%$ with a higher prevalence of $8.4 \%$ in urban areas and lower prevalence of $1.0 \%$ in rural areas [2]. To date several risk factors for GDM are known, including mid-upper arm circumference (MUAC) $\geq 28 \mathrm{~cm}$, family history of type 2 diabetes, previous stillbirth, anaemia, maternal age $>25$ years, $B M I>25[2,4,5]]$, sedentary lifestyle, lower maternal height, cigarette smoking, dietary factors such as sugar-sweetened beverages, high parity, ethnicity (e.g. Asian women tend to be more prone to develop GDM than blacks and whites)[6-8]blood groups, and +Rh factor [9].

Gestational diabetes is associated with a variety of adverse pregnancy outcomes such as macrosomia, dystocia, birth trauma, and metabolic complications in new borns $[10,11]$. Next to that, patients with GDM are known to be at increased risk of developing overt diabetes mellitus (DM) postpartum [9].. Consequently, it is important to identify and manage GDM as early as possible. Currently, in Tanzania GDM is diagnosed from urine by measuring glucose level when mothers attend ante-natal clinic (ANC) regardless of gestational age. However, this is not a reliable method because it has less sensitivity compared to blood glucose test. Universal testing of blood glucose levels may be challenge due to limited resources and capacity in most health facilities in Tanzania $[12,13]$. Therefore, it is essential to develop a simple practical non-invasive tool that is user-friendly at all levels.

Already several studies have been published on the use of risk scores in different settings and population groups, both for the detection of undiagnosed diabetes and for identification of those at future risk $[10,11,14]$. Most of these studies on diabetes risk score have been conducted in Whites and Asian populations and focussed on risk scores for type 2 diabetes mellitus (T2DM) $[2,12,14]$. It is unclear to what extent the findings obtained from the mentioned population can be applied to African settings. Van Leeuwen et al. developed a clinical prediction rule based on patients' characteristics, including family history of diabetes, ethnicity, history of GDM, and body mass index, to identify women at high and low risk for gestational diabetes mellitus early in pregnant Dutch women. The model detected $75 \%$ of women with GDM with a threshold value of $2 \%$ as the predicted probability above which diagnostic testing (OGTT) was performed [11]. Moreover, a study conducted in India 
evaluating the use of an OGTT alone, or in combination with the Indian Diabetes Risk Score showed that use of the risk score followed by OGTT for those at high risk was more efficient and substantially cheaper than using an OGTT alone [15]. In Uganda, 251 pregnant women were screened at 26 to 36 gestational age and hyperglycemia first detected during pregnancy was about $32 \%$ and among them, $23.8 \%$ did not have any risk factor [16]. The authors reported that selective screening may miss up to about $24 \%$ of pregnant women with hyperglycemia [16]. However, there are limited studies on gestational diabetes risk scores; hence the aim of this study was to develop a simple, non-invasive practical tool to predict undiagnosed GDM in Tanzania.

\section{Materials and Methods}

Study setting

This study was conducted using cross-sectional data collected in Dar es Salaam city and Morogoro region from August 2011 to March 2012. Six health facilities in Dar es Salaam (urban) and two health facilities from Kilombero district in Morogoro region (rural) were chosen based on their high number of pregnant women attending ANC. As only $1 \%$ of $6 \%$ of the GDM mothers came from the rural area and $8 \%$ came from the urban area, only data from urban health facilities were used to develop the prediction model [2].

Study population, exclusion and inclusion criteria

Participants were recruited among pregnant women of African ethnicity who were aged $\geq 20$ years, $\geq 20$ gestational weeks, and attending antenatal clinic (ANC) during the survey period. Women with previously diagnosed diabetes were excluded. Other exclusion criteria included the presence of chronic disease(s), such as sickle-cell anaemia or cancer and having conditions that limit activities or normal dietary intake, such as bed-rest since conception. During the ANC visit participants were informed about the purpose of the study, procedure and the possible effects of Oral Glucose Tolerance Test (OGTT), emphasizing that participation was voluntary. In the urban area, 715 mothers were invited, 637 met the criteria for the examination and 599 completed the OGTT with a response rate of $89 \%$. The study protocol was approved by Tanzania National Institute of Medical Research and the respective District Authorities. All participants consented to participate by signing consent forms and few who could not read or write provided oral consent. All information obtained from the participants was confidential and all who were identified with any condition requiring medical attention were referred to medical doctor in charge of the $\mathrm{RCH}$ clinic for further assessment and treatment

Data collection

Physical measurements 
Height was measured without shoes and recorded to the nearest $0.1 \mathrm{~cm}$, using a height measuring board (Shorr productions, Maryland USA). Weight was measured to the nearest $0.1 \mathrm{~kg}$ using Seca Electronic Scale (Seca, Hamburg, Germany). Mid-upper arm circumference (MUAC) was measured using a non-stretchable tape. Pre-gestational weight was assessed at the clinic retrospectively. However, most of the participants came late to the clinic (gestational age ranging from 20 to 38 weeks (mean \pm SD $28 \pm 4.7$ weeks) and only a few participants were able to recall their pre-gestational weight. As MUAC is known to be relatively stable during the pregnancy and highly correlated with pre-pregnancy $\mathrm{BMI}[2,17$, 18], MUAC was used to classify women as normal (MUAC $<28 \mathrm{~cm}$ ) and overweight (MUAC $\geq 28 \mathrm{~cm}$ ). This categorisation lies under the assumption that there was a negligible change of MUAC during pregnancy and that $\mathrm{BMI}=0.1036^{*} \mathrm{MUAC}(\mathrm{mm})-3.9$ as suggested by Khadivzadeh [19]. Systolic and diastolic blood pressures (BP) were measured at the left midupper-arm, while the participant was sitting and relaxed for 10 minutes before the actual measurement, using a digital BP device (Microlife BPA100, Widnau, Switzerland). Blood pressure was measured twice with an interval of 5 to 10 minutes and the average was recorded in millimetre of mercury $(\mathrm{mmHg})$. High blood pressure defined as systolic $\geq 140$ $\mathrm{mmHg}$ and diastolic $\geq 90 \mathrm{mmHg}$ ). Therefore, participants being identified as having high blood pressure were referred to a doctor for further investigations and treatment.

\section{Biochemical assessment}

Participants were asked to collect urine on the spot in a disposable plastic container provided by the research staff. Urine samples were tested within an hour for glucose, ketones, leukocytes and protein using multi-six made with colour sensitive pads (urine strip $10 \mathrm{C}$, Dialeb $\mathrm{GmbH}$, Australia). Overnight fasting blood samples were taken using finger prick with a sterile lancet after cleaning the site with antiseptic alcohol swaps after which blood glucose was measured using HemoCue Glucose B-201 from capillary whole blood. When fasting blood glucose concentrations exceeded the WHO limit for diabetes during the first visit $(n=12)$, participants were asked to return for a second fasting glucose measurement three days later. Participants with normal glucose concentrations were sent for an OGTT test. Anhydrous glucose $(75 \mathrm{~g})$ in $300 \mathrm{ml}$ of water was given and blood glucose was assessed after two hours. Participants were categorized using WHO diagnosis criteria [2], Participants' diagnosed with GDM were referred to the physician for further investigation, treatment and counselling. Haemoglobin level $(\mathrm{Hb})$ was measured using HemoCueHb 201+ Haemoglobin photometer (HemoCue AB, Ängelholm, Sweden) and recorded to the nearest $0.1 \mathrm{~g} / \mathrm{dl}$. Participants were classified as anaemic $(<11 \mathrm{~g} / \mathrm{dl})$ or normal $(\geq 11 \mathrm{~g} / \mathrm{dl})$ using $\mathrm{Hb}$ cutoff points suggested by WHO [20]. They were further classified as severe $(<7 \mathrm{~g} / \mathrm{dl})$, moderate (7-9.9 g/dl), mild (10-10.9 g/dl) and normal ( $\geq 11 \mathrm{~g} / \mathrm{dl})$. 
Demographic data and risk factors were obtained using pre-tested structured questionnaires [2]. , Physical activity was assessed retrospectively using the short form of the International Physical activity Questionnaire (IPAQ revised version 2002) that was translated into Swahili. Participants were asked to recall their activities from the day of the interview up to seven days backward. IPAQ is designed and validated for adults (15-69 years (IPAQ 2005)) and assesses specific types of activity like walking, moderate intensity activities and vigorous intensity activities [21]. Data were reported as metabolic equivalents (METS) according to IPAQ scoring protocol that categorised them as high, moderate and low METS [22] .

\section{Statistical analysis}

Participant characteristics are displayed as mean with SD, median with IQR, or frequencies with percentages. In order to develop the prediction model, we first examined crude associations between predictors - including smoking, alcohol, pre-existing hypertension, birth weight of the previous child, high parity, gravida, previous caesarean section, age, MUAC $\geq 28 \mathrm{~cm}$, previous stillbirth, haemoglobin level, gestational age (weeks), family history of type 2 diabetes, intake of sweetened drinks (soda), physical activity, vegetables and fruits consumption and GDM, using chi-square tests and correlation coefficient for categorical variable and continuous variables, respectively. Potential predictors that were not correlated with GDM were not considered in subsequent analyses, which included smoking, alcohol consumption, pre-existing hypertension, birth weight of the previous child, previous caesarean section, gravida and high parity. Tolerance for all potential predictors was $>0.1$ and Variance Inflation Factor (VIF) was $<10$, indicating that there was no multi-collinearity between the included variables.

Subsequently, binary multivariable logistic regression analysis with stepwise backward selection was used to develop a statistical prediction model for GDM resulting in univariate odds ratios (ORs), 95\% Confidence intervals $(95 \% \mathrm{Cl})$ and $\mathrm{p}$-values. In stepwise regression, a covariate was retained if statistical significance of $15 \%$ was reached. Traditionally, a significance level of $5 \%$ is used to consider the variable to be significant. However, we increased it to $15 \%$ to avoid the inaccurate exclusion of potential predictive variables [23, 24]. To create a risk score, regression coefficient of each significant variable in the multivariable logistic regression analysis was multiplied by 10 and rounded to the nearest integer $[25,26]$. Then, the sum score was calculated for each participant by adding the score for each significant variable in the risk model. The total GDM risk score was calculated as the sum of all individual scores and ranges from 0 to 20. To assess the performance of the prediction model, the receiver operating characteristic (ROC) curve for GDM risk score was built by plotting the sensitivity on the $y$-axis and the false positive rate (1-specificity) on the $x$-axis. Moreover, the area under the curve (AUC) was used to measure the power to discriminate high-risk from low-risk individuals $[14,26]$. The more accurate discriminating the test, the steeper the upward portion of the ROC curve, and the higher the area under 
the curve (AUC). The optimal threshold was determined in achieving the highest sensitivity and specificity with the aim of minimising the number of false negatives. The sensitivity of the model was assessed with respect to the value of the presumed threshold value. The Statistical software program IBM SPSS version 21 was used for statistical analysis.

\section{Results}

Of the 609 participants in the studied population, 8.4\% $(n=51)$ had GDM. Moreover, this population had a mean \pm SD age of $27.5 \pm 5.0$ years gestational age of $28.1 \pm 4.8$ weeks, MUAC of $27.3 \pm 3.8 \mathrm{~cm}$, fasting blood glucose level of $4.6 \pm 1.0 \mathrm{mmol} / \mathrm{L}$, and $2-\mathrm{h}$ glucose (OGTT) of $6.1 \pm 1.1 \mathrm{mmol} / \mathrm{L}$. Most of these participants were moderately physically active $(51.6 \%)$ and completed primary education (69\%). Alcohol consumption (12.6\%) and smoking (1.1\%) during pregnancy was not common in these Tanzanian women (Table 1).

Table 1: Baseline characteristics of women included in this study

\begin{tabular}{ll}
\hline Characteristics & \multicolumn{1}{c}{ Mean \pm SD } \\
\hline Age (years) & $27.5 \pm 5.0$ \\
MUAC (cm) & $27.3 \pm 3.8$ \\
Haemoglobin level (g/dl) & $10.1 \pm 1.6$ \\
Gestational age (weeks) & $28.1 \pm 4.8$ \\
Fasting blood glucose (mmol/L) & $4.6 \pm 1.0$ \\
2-h glucose (OGTT) (mmol/L) & $6.1 \pm 1.1$ \\
& $\mathrm{n}(\%)$ \\
Previous stillbirth, $\mathrm{n}$ yes (\%) & $93(15.3)$ \\
Family history of type 2 diabetes, $\mathrm{n}$ yes (\%) & $88(14.4)$ \\
Intake coffee, tea, other beverages, $\mathrm{n}$ yes (\%) & $520(85.4)$ \\
Vegetable consumption, $\mathrm{n}$ yes (\%) & $212(34.8)$ \\
Fruits consumption, $\mathrm{n}$ yes (\%) & $107(17.6)$ \\
Alcohol consumption, $\mathrm{n}$ yes (\%) & $77(12.6)$ \\
Smokers, $\mathrm{n}$ yes (\%) & $7(1.1)$ \\
Marital status, $\mathrm{n}$ Married (\%) & $507(83.3)$ \\
Single & $61(10.0)$ \\
Other (widowed, cohabiting \& separated) & $41(6.7)$ \\
Level of physical activity & \\
Low & $124(20.4)$ \\
Moderate & $314(51.6)$ \\
High & $171(28.0)$
\end{tabular}




$\begin{array}{ll}\text { Level of education } & 38(6.2) \\ \text { Informal } & 420(69.0) \\ \text { Primary } & 131(21.5) \\ \text { Secondary } & 20(3.3) \\ \text { Post-secondary } & \\ \text { Source of income } & 293(48.1) \\ \text { House works } & 104(17.1) \\ \text { Salary/wage } & 209(34.3) \\ \text { Petty business } & 3(0.5) \\ \text { Agriculture } & \end{array}$

The results of the univariate logistic regression model are shown in table 2, including potential predictors for the GDM model and their OR $(95 \% \mathrm{Cl})$, coefficients $(\beta)$ and $p$-value. MUAC $\geq 28$, previous stillbirth, family history of type 2 diabetes, haemoglobin level, and fruits and vegetable consumption, were associated with GDM with $p=$ value 0.15 .

Table 2: Potential predictors of GDM and their Odds ratios, coefficients and p-value.

\begin{tabular}{|c|c|c|c|}
\hline & \multicolumn{3}{|c|}{ Univariate model: $n=51$} \\
\hline & \multicolumn{3}{|c|}{ n of whom who had GDM } \\
\hline & $\mathrm{OR}^{\mathrm{a}}(95 \% \mathrm{Cl})^{\mathrm{b}}$ & $\beta^{c}$ & $P^{d}$ \\
\hline \multicolumn{4}{|l|}{ Intercept } \\
\hline Age (years) & 1.01 (0.95-1.07) & 0.01 & 0.78 \\
\hline MUAC $\geq 28 \mathrm{~cm}$ & $1.78(0.99-3.18)$ & 0.58 & 0.051 \\
\hline Previous stillbirth & $2.56(1.34-4.90)$ & 0.94 & 0.004 \\
\hline HB & $0.80(0.67-0.96)$ & -0.22 & 0.014 \\
\hline Gestational age (weeks) & $0.98(0.92-1.04)$ & -0.02 & 0.505 \\
\hline Family history of type 2 diabetes & $2.20(1.12-4.33)$ & 0.79 & 0.022 \\
\hline $\begin{array}{l}\text { Sweetened drinks (soda) } \\
\text { consumption }\end{array}$ & $0.78(0.37-1.67)$ & -0.25 & 0.523 \\
\hline Vegetable consumption & $1.75(0.98-3.12)$ & 0.56 & 0.058 \\
\hline Physical activity & $1.00(1.00-1.00)$ & 0.00 & 0.573 \\
\hline Fruits consumption & 1.69 (0.87-3.29) & 0.52 & 0.124 \\
\hline
\end{tabular}


Table 3 presents the results from a multivariate model, including the ORs, B's, and risk scores for the non-invasive characteristics $M U A C \geq 28$, previous stillbirth, and family history of type 2 diabetes. Fruits and vegetable consumption were removed from the model by backward elimination method.

Table 1 Risk variables factors in the multivariate logistic model

\section{Multivariate model ( 51 GDM cases) Score}

\begin{tabular}{llllll} 
Variables & $\mathbf{O R}^{\mathbf{a}}(\mathbf{9 5 \%} \mathbf{C l})^{\mathbf{b}}$ & $\mathbf{B}^{\mathbf{c}}$ & $\mathbf{P}^{\mathbf{d}}$ & $\left.\mathbf{( \beta}^{* 10}\right)$ \\
\hline Intercept & 0.056 & -2.89 & 0 & \\
MUAC $\geq 28 \mathrm{~cm}$ & $1.66(0.92-2.99)$ & 0.51 & 0.095 & 5 \\
Previous still birth (yes) & $2.42(1.26-4.67)$ & 0.89 & 0.008 & 9 \\
Family history of type 2 diabetes (yes) & $1.87(0.93-3.73)$ & 0.62 & 0.077 & 6 \\
Area under the ROC curve & & & & 0.64
\end{tabular}

${ }^{a}=$ Odds ratios, ${ }^{b}=95 \%$ confidence interval, ${ }^{c} \log$ OR/ coefficient and ${ }^{d}=$ Significance level.

As higher risk scores - ranging from 0-20 points - indicate greater weight to a respective risk, previous stillbirth was identified as being a more important predictor for GDM than MUAC. The performance of the predictive risk score/tool is shown in figure 1 . The area under the ROC curve (AUC) for the developed risk score is $0.640(95 \% \mathrm{Cl} ; 0.56-0.72 ; \mathrm{p} 0.001)$, which indicates that the developed score is able to correctly classify $64 \%$ of high-risk individuals.

The GDM risk score value of 2.5 was selected as the cut-off point for increased risk of undiagnosed GDM, along with the sensitivity of 0.69 and specificity of 0.53 , positive predictive value (PPV) of $12 \%$ and negative predictive value (NPV) of $95 \%$. This cut-off value was chosen to maximise sensitivity and specificity, aiming to minimise the number of undiagnosed GDM (false negatives). Moreover, comparison of two threshold values in table 4 shows that $31 \%$ of mothers will remain undiagnosed with a cut-off value of 2.5 . When changing the cut-off value to 5.5 this increases up to $55 \%$ of mothers remaining undiagnosed (false negatives), which even further increases as we change the cut-off value to 7.5 or 10 .

Table 2: comparison of the threshold values for the risk of GDM

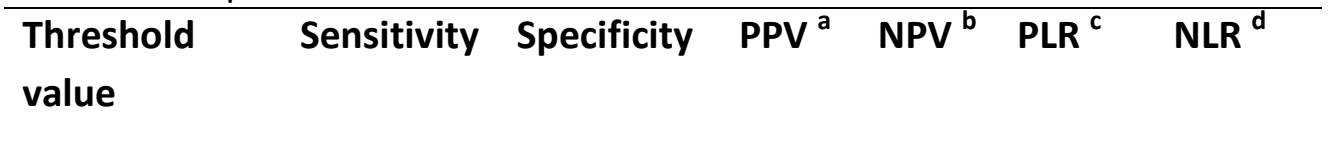




\begin{tabular}{lllllll}
\hline 2.5 & 0.69 & 0.53 & 0.12 & 0.95 & 1.47 & 0.58 \\
5.5 & 0.45 & 0.75 & 0.14 & 0.94 & 1.8 & 0.73 \\
7.5 & 0.37 & 0.81 & 0.15 & 0.93 & 1.95 & 0.78 \\
10.0 & 0.26 & 0.89 & 0.18 & 0.94 & 2.36 & 0.83
\end{tabular}

$\bar{a}=$ positive predictive value, ${ }^{b}=$ negative predictive value, ${ }^{c}=$ positive likelihood ratio, ${ }^{d}=$ negative likelihood ratio.

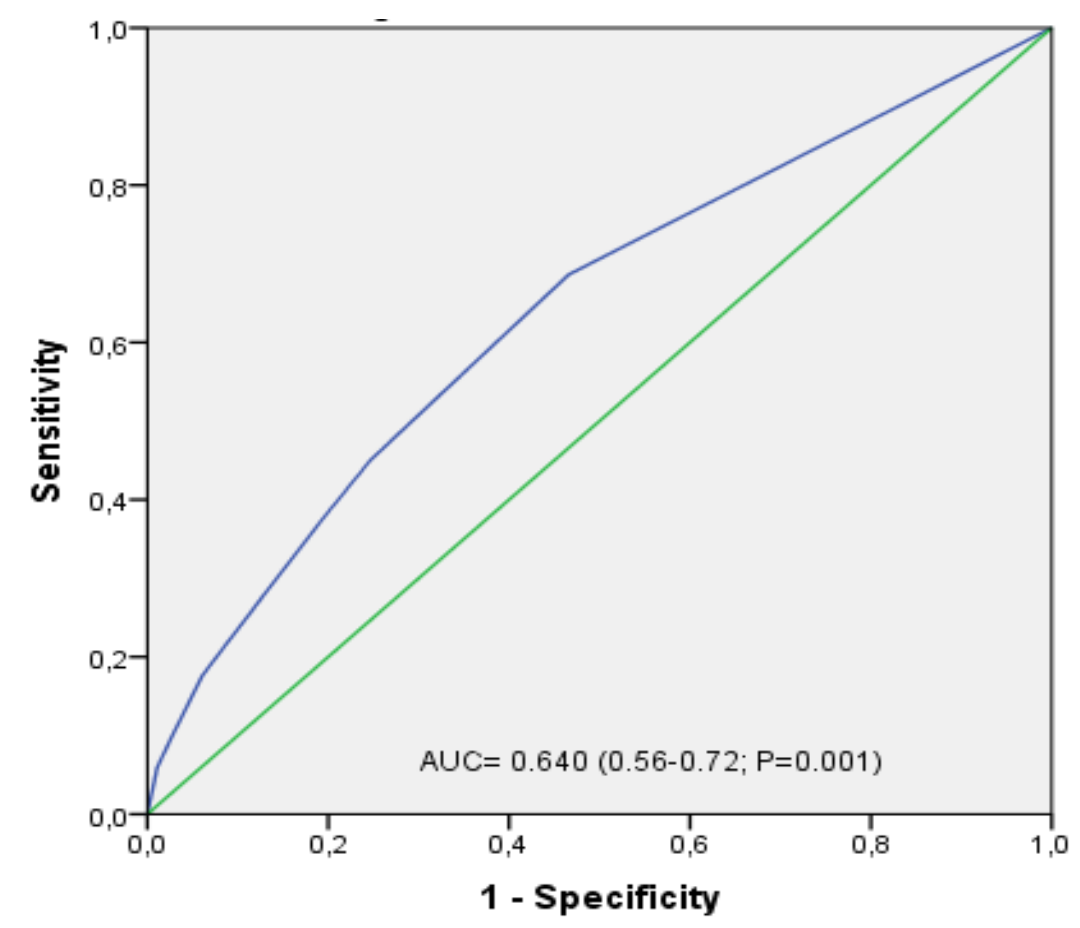

Figure 1 Shows the ROC curve for the GDM risk score in derivation population.

\section{Discussion}

A simple non-biochemical score could play an important role as the first step in the process of identifying individuals with an increased likelihood of having prevalent but undiagnosed diabetes [26], particularly in low-income countries. Therefore, the main goal of this study was to develop a simple, non-invasive, practical tool to predict undiagnosed GDM in Tanzanian women. A multivariate model was used to develop the tool, including MUAC, previous stillbirth, and family history of type 2 diabetes. With this model, 69\% of the GDM cases presenting at various ANC's in Tanzania were identified. At the same time, we were able to identify $53 \%$ of the none-GDM cases presented in those ANC's. Moreover, with an 
AUC of $0.64(0.56-0.72)$ and a threshold value of $2.5,6$ out of every 10 high risks individuals could be correctly identified.

Our results are in line with the findings from van Leeuwen et.al who developed a clinical screening tool to identify women at high and low risk for GDM early in pregnant Dutch women. In their study, family history of diabetes, ethnicity, history of GDM, and body mass index were shown to be important predictors of GDM, which detected $75 \%$ of women with GDM with a threshold value of $2 \%$ as the predicted probability above which diagnostic testing (OGTT) was performed [11].Additionally, Naylor et.al developed a clinical scoring system based on age, BMI before pregnancy, and race from a prospective cohort study [7]. Their scoring system reduced the number a screening test performed by $34.6 \%$ and detected $81.2 \%$ to $82.6 \%$ of the women with GDM as compared with $78.3 \%$ classified during usual care implying that the use of women's clinical characteristics allows efficient selective screening for gestational diabetes. Furthermore, data of a Turkish retrospective case-control study showed that maternal age, BMI, first-degree relatives with a diagnosis of diabetes mellitus, prior macrosomic foetus, and adverse outcome during the previous pregnancies were the most important predictors of GDM which detected $85 \%$ of cases with GDM [7, 16, 27]. Unlike these other studies age of participants and gestational age were not significantly associated with GDM risk in our study. Probably this is because teenagers were excluded and we screened women regardless of gestational age ranging from 20 to 38 weeks. In Uganda, out of 80 (31.9\%) pregnant women with hyperglycemia in pregnancy, 9 (23.8)\% did not have any risk factor [16] \{Caliskan, $2004 \# 4\}$.

Based on the above summarized literature it can be concluded that our GDM risk score did not perform as good as the risk score calculated in the study by van Leeuwen et.al [11]], and several of the other studies $[16,27]$. This difference may be explained by the inclusion of different variables, for example, van Leeuwen et al included pre-gestational BMI in their model. Unfortunately, we were not able to test the impact of pre-gestational BMI as most participants did not remember their pre-gestational weight at the time they reported at the ANC (gestational age 8-32 weeks, with a mean of 20 weeks). However, we did include MUAC, which is easy to measure and has been shown to be a relatively stable measure during pregnancy and $[19,28]$. In addition, van Leeuwen et al identified a history of GDM as a significant predictor of GDM. Unfortunately, history of GDM was not assessed in our study. Another reason for the difference between our score and the score developed by van Leeuwen et al could be that we studied an African population while Van Leeuwen studied a Western population. In addition, due to cross-sectional nature of the current study, it is possible that some women could have been diagnosed with GDM if further screening had been done between 24 and 28 weeks to those who were negative at earlier gestational age. For example, $\sim 18 \%$ of our participants had gestational age $<24$ weeks and $\sim 84 \%$ of them 
were negative. This may have resulted in misclassification of participants and consequently may affect the sensitivity of our study. Apart from pre-gestational BMI incorporated in a study by van Leeuwen et.al, the age of the participants, gestational age and pre-gestational BMI were also included into the model built by Naylor et.al and Caliskan et. al as significant risk factors in their population which were not risk factors in our study probably because we excluded teenagers \{Caliskan, 2004 \#4;Naylor, 1997 \#19\}. These could also explain the low performance of our model.

Thus, overall, these results may not seem that promising. However, it is important to note that those identified by the risk score (true positives) tend to be at higher risk of micro and macrovascular complications than those who are missed (false negatives) [15, 29]. Some of them might also be hyperglycemia detected first time during pregnancy. Although our screener is not very sensitive, we think it is an improvement of the current situation since it is important to identify more of the people at risk given the major possible complications. Nevertheless, it is important to understand the risks of adverse outcomes in the significant minority of individuals with undiagnosed GDM (false negatives), which would be $31 \%$ based on our analyses.

When interpreting our results, several aspects need to be considered. This is the first study to develop GDM risk score for GDM in Tanzania and in is among the few ones in Africa. Moreover, we feel that the sample size was adequate for the prediction model. As a rule of thumb, there should be 10 events per variable $[24,29]$. In this study, we had 3 predictor variables in the final model, which requires at least 30 GDM cases where we had 51 GDM cases. Furthermore, due to the fact that we only used data from urban ANCs to develop our screening tool, we did not have to control for known urban/rural risk modifiers as education level and occupation level. However, there are also some limitations of this study. Misclassification of participants especially those who were screened at gestational age $<24$ weeks and found negative. This might influence the sensitivity of our study. Moreover, some variables, including physical activity, and pre-gestational weight, were assessed retrospectively, which may have been a source of bias as well. Finally, due to practical reasons that are the aim to develop a non-invasive tool - haemoglobin level was excluded from the multivariate model, which reduced model performance. Lastly, there is a need to recalibrate the developed score before actual implementation. This can be done in a disease incidence and risk factor distribution of the target population where data from National database and hospital or general practitioners can be used [30]. Studies show that most of the original models perform better when recalibrated [30]. Brown et al. reported a rapid increase in the number of newly developed risk scores with similar variables but sometimes perform poorly upon external validation [10]. Therefore, we emphasize the need for recalibration, validation and applicability of those existing scores to other settings $[10,30]$

\section{Conclusion and recommendation for future research}


The findings of this study indicate that MUAC, previous stillbirth and family history of type 2 diabetes are significant risks factors for the development of GDM in Tanzanian population. However, the developed score $(A \cup C=0.640(0.56-0.72))$ did not perform well in distinguishing those with GDM risk from those without GDM risk. Despite this, a simple, non-invasive practical tool, if well-developed, might be a useful tool to predict undiagnosed GDM especially in developing countries where there is a lack of protocol for GDM screening, qualified personnel, and screening tools specifically in Sub-Saharan Africa setting. We recommend further studies on improving the model, for example by including pregestational BMI and pregnancy weight gain. Therefore, recalibration, validation and applicability of the current score to another setting are vital in the future studies.

\section{Acknowledgements}

We would like to thank the Netherlands University Foundation for International Cooperation (NUFFIC) for the support in carrying out the study. We acknowledge support from respective district authorities, research assistants, health workers from the surveyed health facilities for their cooperation and the mothers who accepted to participate in the study.

\section{Conflict of interests}

The authors have declared no conflict of interest. 


\section{Reference}

1. Chu SY, Callaghan WM, Kim SY, Schmid CH, Lau J, England L, Dietz PM: Maternal obesity and risk of gestational diabetes mellitus. Diabetes care 2007, 30(8):20702076.

2. Mwanri AW, Kinabo J, Ramaiya K, Feskens EJ: Prevalence of gestational diabetes mellitus in urban and rural Tanzania. Diabetes research and clinical practice 2014, 103(1):71-78.

3. Xiong $X$, Saunders L, Wang F, Demianczuk N: Gestational diabetes mellitus: prevalence, risk factors, maternal and infant outcomes. International Journal of Gynecology \& Obstetrics 2001, 75(3):221-228.

4. Radesky JS, Oken E, Rifas-Shiman SL, Kleinman KP, Rich-Edwards JW, Gillman MW: Diet during early pregnancy and development of gestational diabetes. Paediatric and perinatal epidemiology 2008, 22(1):47-59.

5. Shirazian N, Emdadi R, Mahboubi M, Motevallian A, Fazel-Sarjuei Z, Sedighpour N, Fadaki S-F, Shahmoradi N: Screening for gestational diabetes: usefulness of clinical risk factors. Archives of gynecology and obstetrics 2009, 280(6):933-937.

6. Hod M, Kapur A, Sacks DA, Hadar E, Agarwal M, Di Renzo GC, Roura LC, McIntyre HD, Morris JL, Divakar H: The International Federation of Gynecology and Obstetrics (FIGO) Initiative on gestational diabetes mellitus: A pragmatic guide for diagnosis, management, and care. International journal of gynaecology and obstetrics: the official organ of the International Federation of Gynaecology and Obstetrics 2015, 131:S173.

7. Naylor CD, Sermer M, Chen E, Farine D: Selective screening for gestational diabetes mellitus. New England Journal of Medicine 1997, 337(22):1591-1596.

8. Yang X, Hsu-Hage B, Zhang H, Yu L, Dong L, Li J, Shao P, Zhang C: Gestational diabetes mellitus in women of single gravidity in Tianjin City, China. Diabetes Care 2002, 25(5):847-851.

9. Karagoz H, Erden A, Ozerhan Ozer KE, Cetinkaya A, Avci D, Karahan S, Basak M, Bulut $\mathrm{K}$, Mutlu $\mathrm{H}$, Simsek $\mathrm{Y}$ : The role of blood groups in the development of diabetes mellitus after gestational diabetes mellitus. Therapeutics and clinical risk management 2015, 11:1613.

10. Brown N, Critchley J, Bogowicz P, Mayige M, Unwin N: Risk scores based on selfreported or available clinical data to detect undiagnosed type 2 diabetes: a systematic review. Diabetes research and clinical practice 2012, 98(3):369-385.

11. Van Leeuwen $M$, Opmeer $B$, Zweers $E$, van Ballegooie $E$, ter Brugge $H$, De Valk $H$, Visser G, Mol B: Estimating the risk of gestational diabetes mellitus: a clinical prediction model based on patient characteristics and medical history. BJOG: An International Journal of Obstetrics \& Gynaecology 2010, 117(1):69-75.

12. Magoma M, Requejo J, Merialdi M, Campbell OM, Cousens S, Filippi V: How much time is available for antenatal care consultations? Assessment of the quality of care in rural Tanzania. BMC Pregnancy and Childbirth 2011, 11(1):64.

13. Gross K, Armstrong Schellenberg J, Kessy F, Pfeiffer C, Obrist B: Antenatal care in practice: an exploratory study in antenatal care clinics in the Kilombero Valley, south-eastern Tanzania. BMC Pregnancy Childbirth 2011, 11:36.

14. Lindström J, Tuomilehto J: The Diabetes Risk Score A practical tool to predict type 2 diabetes risk. Diabetes care 2003, 26(3):725-731. 
15. Mohan V, Goldhaber-Fiebert JD, Radha V, Gokulakrishnan K: Screening with OGTT alone or in combination with the Indian diabetes risk score or genotyping of TCF7L2 to detect undiagnosed type 2 diabetes in Asian Indians. The Indian journal of medical research 2011, 133(3):294.

16. Nakabuye B, Bahendeka S, Byaruhanga R: Prevalence of hyperglycaemia first detected during pregnancy and subsequent obstetric outcomes at St. Francis Hospital Nsambya. BMC Research Notes 2017, 10:174.

17. Gale CR, Javaid MK, Robinson SM, Law CM, Godfrey KM, Cooper C: Maternal size in pregnancy and body composition in children. The Journal of Clinical Endocrinology \& Metabolism 2007, 92(10):3904-3911.

18. Ricalde AE, Velásquez-Meléndez G, Tanaka ACdA, de Siqueira AA: Mid-upper arm circumference in pregnant women and its relation to birth weight. Revista de Saúde Pública 1998, 32(2):112-117.

19. Khadivzadeh T: Mid upper arm and calf circumferences as indicators of nutritional status in women of reproductive age. 2002.

20. WHO: Haemoglobin concentrations for the diagnosis of anemia and assessment of severity. In: Vitamin and Mineral Nutrition Information System. Edited by Organization WH. Geneva; 2011.

21. IPAQ: International Physical Activity Questionnaire. In. http//wwwipaqkise/ipaqhtm.; 2002.

22. IPAQ: Guidelines for Data Processing and Analysis of the International Physical Activity Questionnaire In. http://wwwipaqkise/scoringpdf.; 2005.

23. Bursac Z, Gauss CH, Williams DK, Hosmer DW: Source Code for Biology and Medicine. Source code for biology and medicine 2008, 3:17.

24. Moons KG, Kengne AP, Woodward M, Royston P, Vergouwe $Y$, Altman DG, Grobbee DE: Risk prediction models: I. Development, internal validation, and assessing the incremental value of a new (bio) marker. Heart 2012, 98(9):683-690.

25. De Sousa AGP, Pereira AC, Marquezine GF, do Nascimento-Neto RM, Freitas SN, Nicolato RLdC, Machado-Coelho GLL, Rodrigues SL, Mill JG, Krieger JE: Derivation and external validation of a simple prediction model for the diagnosis of type 2 diabetes mellitus in the Brazilian urban population. European journal of epidemiology 2009, 24(2):101-109.

26. Ramachandran A, Snehalatha C, Vijay V, Wareham N, Colagiuri S: Derivation and validation of diabetes risk score for urban Asian Indians. Diabetes research and clinical practice 2005, 70(1):63-70.

27. Caliskan E, Kayikcioglu F, Öztürk N, Koc S, Haberal A: A population-based risk factor scoring will decrease unnecessary testing for the diagnosis of gestational diabetes mellitus. Acta obstetricia et gynecologica Scandinavica 2004, 83(6):524-530.

28. Okereke C, Anyaehie U, Dim C, lyare E, Nwagha U: Evaluation of some anthropometric indices for the diagnosis of obesity in pregnancy in Nigeria: a crosssectional study. African health sciences 2014, 13(4):1034-1040.

29. Mayige M: Derivation and validation of a simple risk score for undiagnosed diabetes for Tanzania and other African populations. 2014.

30. van der Leeuw J, van Dieren S, Beulens J, Boeing H, Spijkerman A, van der Graaf $Y$, Nöthlings $U$, Visseren F, Rutten G, Moons K: The validation of cardiovascular risk scores for patients with type 2 diabetes mellitus. Heart 2015, 101(3):222-229. 
